\title{
FETAL OUTCOME AFTER CAESAREAN SECTION PERFORMED FOR FETAL DISTRESS BASED ON ABNORMAL CARDIOTOCOGRAPHY
}

\author{
Shahzadi Saima Hussain, Syeda Sitwat Fatima, Tanveer Shafqat, Faiza Rasool \\ Department of Gynaecology Lady Read Reading Hospital, Peshawar - Pakistan
}

\begin{abstract}
Objective: To determine the fetal outcome after Caesarean Sections (CS) performed for fetal distress (FD) based on Cardiotocography (CTG) along with fetal outcome.

Material and Methods: This retrospective chart review was conducted at the Gynaecology and Obstetrics Department of Lady Reading Hospital Peshawar from June 2015 to June 2016 after approval from hospital ethical committee, using non-probability convenient sampling technique. Hospital record of 234 patients who had CS for fetal distress was reviewed. Patients with singleton term gestation who had CS for fetal distress diagnosed on the basis of abnormal Cardiotocography alone were analyzed. Fetal outcome was noted in terms of Apgar score at $5 \mathrm{~min}$, admission to neonatal intensive care unit (NICU) and perinatal mortality. The results are expressed in frequencies and percentages and shown in tables and charts.
\end{abstract}

Results: In one year, total 1255 CS were performed, among them 234 (18.64\%) fulfilling inclusion criteria were enrolled. Babies delivered with 5 minute Apgar score of 7 or above were 166 (70.94\%). Twenty-one (8.97\%) babies were admitted in Neonatal ICU and perinatal mortality was 6 (2.54\%).

Conclusion:Fetal Distress diagnosed on CTG alone poorly correlates with fetal outcome. In 70.94\% of Caesarean sections, fetal outcome was good. These CS could have been prevented by restricting CTG to high risk patients, availability of fetal blood sampling and confirmation of diagnosis by cord ABG's and $\mathrm{PH}$.

Key words: Fetal Distress, CTG, Caesarean section.

This article may be cited as: Hussain SS, Fatima SS, Shafqat T, Rasool F. Fetal outcome after Caesarean Section performed for fetal distress based on abnormal Cardiotocography. J Med Sci 2021 January;29(1):13-16

\section{INTRODUCTION}

Fetal distress (FD) is characterized by abnormalities in fetal heart rate. This distress is usually due to temporary or permanent impairment of oxygen supply to the fetus resulting in hypoxia or acidosis. Fetal hypoxia is a common cause of fetal mortality and morbidity ${ }^{1-3}$. Fetal hypoxia leads to metabolic acidosis resulting in accumulation of hydrogen ion, which causes a low $\mathrm{PH}$ in fetal blood. This in turn causes changes in fetal heart pattern which can be observed on Cardiotocography (CTG). Intermittent fetal heart rate (FHR) auscultation and CTG monitoring are the most common methods of intrapartum fetal monitoring. Though, a normal trace is $98 \%$ predictive of a normal acid base balance at birth but an abnormal CTG trace has a low predictive value of adverse fetal outcome (30\%), the false-positive rate of the CTG is around $60 \%$ which often

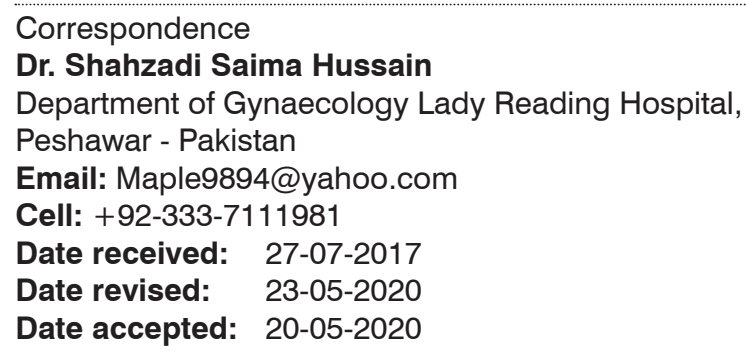

results in unnecessary Casarean section (CS) ${ }^{3-7}$.

Suspected FD diagnosed on CTG is one of the leading indications for CS for past few decades. As diagnosis of fetal distress on CTG alone has resulted in increase in CS rate, it should be coupled with FBS. FBS is a useful tool for diagnosis of fetal hypoxia and acidosis. FBS $\mathrm{pH}$ value of less than 7.20 has a higher specificity than a pathological CTG to predict low Apgar score. Along with this, umbilical cord blood gas analysis is highly recommended in all high-risk deliveries where fetal hypoxia is suspected. It has its own clinical and medico legal significance ${ }^{8-13}$. CS carries the risk of maternal mortality and morbidity, in both current and future pregnancies. High cost of CS adds tremendously to financial burden on health system in developing countries. Avoiding caesarean section where possible will help in decreasing adverse pregnancy outcomes in next pregnancy too. In this context this study was conducted to critically assess CS performed for FD considering fetal outcome ${ }^{14,15}$.

\section{MATERIAL AND METHODS}

This retrospective chart review was conducted in one of the Gynaecology and Obstetrics unit of Lady Reading hospital in Peshawar from June 2015 to June 2016. A 
Fetal Outcome After Caesarean Section Performed For Fetal Distress Based On Abnormal Cardiotocography.

total 1255 CS were performed. Among them, 321 (23.69\%) patients had CS for fetal distress. Using non-probability Convenience sampling, 234 ( 18.64\%) were included in this study as they fulfilled inclusion criteria. These were the patients with single alive intrauterine pregnancy with term gestation $(37+1-41+6$, calculated by 1 st trimester scan or LMP) and no fetal abnormality on ultrasound who underwent CS for suspicious or pathological CTG (CTG interpreted as suspicious or pathological according to NICE Guidelines on intrapartum care) ${ }^{14}$. Patients with antepartum hemorrhage, chorioamnionitis, intrauterine growth restriction, eclampsia, mal-presentation and with more than one previous CS were excluded from study. The primary outcome of study was neonatal and perinatal outcome. Neonatal outcome was defined as Apgar score at $5 \mathrm{~min}$, (Apgar score of 7-10 was considered reassuring, 4-6 as moderately abnormal and score of $0-3$ as low in term baby) and NICU admission and perinatal outcome in terms of mortality within 7 days of birth ${ }^{16}$. It was noted from patient record whether the fetal blood sampling and post natal umbilical cord arterial blood gas analysis was carried out or not. The results are expressed in frequencies and percentages and presented in form of tables and charts.

\section{RESULTS}

This study showed that majority of babies were born healthy with good Apgar score, despite being diagnosed to have abnormal CTG (figure 1), meconium was noted in almost half of the cases (Table 1) but had no adverse effect on neonatal outcome (figure 2 and 3 ). Ninety-one percent $(n=213)$ of babies went home without requiring neonatal ICU admission (Fig 2). Among those admitted, 16 babies were discharged within 24-48 hours, 2 were treated for meconium aspiration syndrome, 3 babies died in nursery while 3 were fresh still birth. In total, $2.54 \%$ (6) of babies died within 1 week of life (early neonatal death).

Table 1: Meconium Stained Liqor (MSL)

\begin{tabular}{|l|c|c|c|}
\hline MSL present & $\mathbf{5 1 . 7 0 \% (} \mathbf{n = 1 2 1})$ & Grade 1 & 9.91\% $(\mathrm{n}=12)$ \\
\cline { 3 - 4 } & & Grade 2 & $33.88 \%(\mathrm{n}=41)$ \\
\cline { 3 - 4 } & & Grade 3 & $43.01 \%(\mathrm{n}=53)$ \\
\hline MSL Absent & $48.29 \%(\mathrm{n}=113)$ & & \\
\hline
\end{tabular}

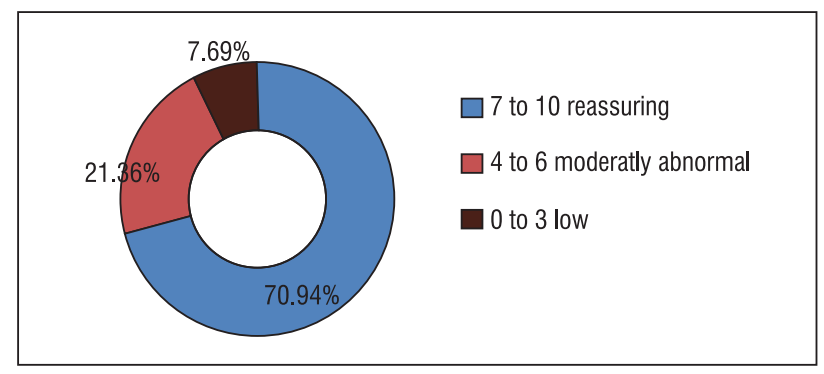

Fig 1: Apgar score at $5 \mathrm{~min}$

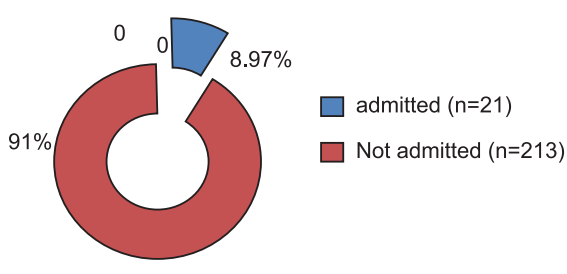

Fig 2: NICU admission.

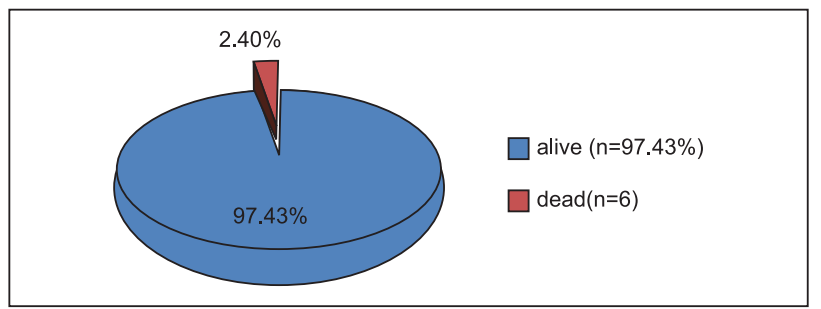

Fig 3: Perinatal outcome.

DISCUSSION

CTG has good positive predictive value when it is used as a screening test to indicate non-compromised condition of fetus. Its use and importance in this aspect cannot be denied, but its ubiquitous presence in labor room has led to its injudicious use in low risk woman, its complicated interpretation and fear of litigation of compromised babies has led to low threshold and increased rate of CS.

Use of CTG alone to diagnose fetal distress correlates poorly with fetal outcome In this study, $70.94 \%$ babies were born with reassuring Apgar score, after they had CS performed for fetal distress. A Cochrane review published in 2017 concluded that 'CTG during labor is associated with reduced rates of neonatal seizures, but no clear differences in cerebral palsy, infant mortality or other standard measures of neonatal wellbeing. However, its use increased the rate of instrumental and caesarean deliveries significantly ${ }^{17,18}$.

In low resource countries like Pakistan, unfortunately still it is CTG alone, on which fetal distress is diagnosed and managed. In this study, the rate of CS performed for FD based on abnormal CTG findings was $18.64 \%(n=234)$. A study in Bangladesh reported $21 \%$ caesarean section rate for FD and another local study reported it as $27 \% 18,19,22$.

Our study revealed that $8.97 \%(n=21)$ of neonates were admitted in Neonatal ICU. Among them,$^{18}$ were treated and discharged from Neonatal ICU. Roy KK at el noted $15.2 \% \mathrm{NICU}$ admissions in a related study while perinatal mortality was $2.54 \%$, which is comparable to $3.1 \%$ perinatal mortality in other similar studies ${ }^{9,22}$.

Meconium stained liquor (MSN) is considered as sign of fetal distress. In spite of presence of meconium, fetal outcome was reassuring in majority of cases $(70.94 \%)$, 
Fetal Outcome After Caesarean Section Performed For Fetal Distress Based On Abnormal Cardiotocography.

which shows that decision of immediate delivery should not be based on presence of meconium only. In our study, it was present in $51.70 \%$ of patients along with non-reassuring FHR, which is comparable to another study which reported it in $49 \%$ of cases. An other similar study reported CS in presence of MSL in $42.04 \%^{22-24}$.

Other methods like FBS should be performed in association with electronic fetal monitoring patterns, where abnormality is noted. There is only one RCT comparing CS rate for fetal distress in CTG alone with combination of CTG and FBS where the results were $18 \%$ versus $11 \%$ in later group. Fetal pulse oximetry is another modality which can be utilized to confirm CTG based diagnosis of fetal distress. It is less invasive and seems as reliable as $\mathrm{FBS}^{25-30}$. Umbilical cord blood sampling should be carried out in all high-risk deliveries. It gives insight into etiology of asphyxia as well as it confirms or negates antenatal fetal compromise and justification of CS for fetal distress ${ }^{9,10}$. Keeping in view the impact, consequences and financial burden of CS, these modalities should be explored, which can lead to significant decrease in unnecessary CS rate for fetal distress.

\section{CONCLUSION}

Use of CTG alone to diagnose fetal distress can lead to unnecessary operative intervention. In an environment of poor health seeking behavior, the future of these women will be in jeopardy as many of them may avoid skilled birth attendants in their subsequent deliveries with its serious consequences, like repeat CS, uterine rupture and postpartum haemorrhage ${ }^{31,32}$. Its use should continue to be investigated by carefully designed randomized controlled trials to optimize its utility and compare outcomes with newer, potentially more accurate ways of evaluating fetal well-being in labor.

\section{REFERENCES}

1. Mosby's medical dictionary. 9th edition; 2009, St. Louis, MO; mosby Elsevier.

2. Velayudhareddy S, Kirankumar H. Management of fetal asphyxia by intrauterine fetal resuscitation. Ind $\mathrm{J}$ anaesthe. 2010 Sep;54(5):394.

3. Hutter D, Jaeggi E. Causes and mechanisms of intrauterine hypoxia and its impact on the fetal cardiovascular system: a review. Intern J pedia. 2010 Oct 19;2010.

4. ACOG Practice Bulletin. No. 62: Intrapartum fetal heart rate monitoring. Obstet Gynecol. 2005 May;105(5 Pt 1):1161-9.

5. Beard RW, Filshie GM, Knight CA, Roberts GM. The significance of the changes in the continuous fetal heart rate in the first stage of labour. BJOG: An Intern J Obstetr \& Gynaec. 1971 Oct 1;78(10):865-81 .

6. Hubaishi NM, Cherifi F, Taka MK, Santosh A. Justified indicators for caesarean section in fetal distress. Hamdan
Med J. 2013 Mar;6(1):79-83.

7. Pinas A, Chandraharan E. Continuous cardiotocography during labour: Analysis, classification and management. Best practice \& research Clini obstetr \& gynaeco. 2016 Jan 1;30:33-47.

8. Ayres-de-Campos . Introduction: why is intrapartum foetal monitoring necessary-impact on outcomes and interventions. Best Practice \& Resea Clini Obstetr \& Gynaeco. 2016 Jan 1;30:3-8.

9. Roy KK, Baruah J, Kumar S, Deorari AK, Sharma JB, Karmakar D. Cesarean section for suspected fetal distress, continuous fetal heart monitoring and decision to delivery time. The Ind J Pediatr. 2008 Dec 1;75(12):1249-52.

10. Spencer JA. Clinical overview of cardiotocography. BJOG: Intern J Obstetr \& Gynaec. 1993 Mar 1;100(s9):47.

11. Mahendru AA, Lees CC. Is intrapartum fetal blood sampling a gold standard diagnostic tool for fetal distress?. European J Obstetr \& Gynec and Repro Biology. 2011 Jun 30;156(2):137-9.

12. Thomas J, Paranjothy S. Royal college of Obstetricians and Gynaecologists. Clini Effective. 2001.

13. ACOG Committee on Obstetric Practice. ACOG Committee Opinion No. 348, November 2006: Umbilical cord blood gas and acid-base analysis. Obstetr and gynec. 2006 Nov;108(5):1319.

14. Petitti DB. Maternal mortality and morbidity in cesarean section. Clin Obstetr and Gynec. 1985 Dec 1;28(4):7639.

15. Mahmood A, Sultan M. National Institute of Population Studies (NIPS)(Pakistan), and Macro International Inc. Pak Demogra and Health Survey. 2006;7:123-45.

16. Nunes VD, Gholitabar M, Sims JM, Bewley S. Guidelines: Intrapartum care of healthy women and their babies: summary of updated NICE guidance. The BMJ. 2014;349.

17. Alfirevic Z, Devane D, Gyte GM, Cuthbert A. Continuous cardiotocography (CTG) as a form of electronic fetal monitoring (EFM) for fetal assessment during labour. Cochrane Database Syst Rev 2017;2:CD006066.

18. Intrapartum care: care of healthy women and their babies during childbirth; NICE Clinical Guideline (Dec 2014)

19. Shrestha M, Shrestha S. Cesarean Section profile at a tertiary center. Nepal J Obstetr and Gynaec. 2020 Jun 7;15(1):68-71.

20. American Academy of Pediatrics. Neonatal encephalopathy and neurologic outcome. Pediatrics. 2014 May 1;133(5):e1482-8.

21. Tasnim N, Mahmud G, Akram S. Predictive accuracy of intrapartum cardiotocography in terms of fetal acid base status at birth. J Coll Physicians Surg Pak. 2009 Oct $1 ; 19(10): 632-5$. 
Fetal Outcome After Caesarean Section Performed For Fetal Distress Based On Abnormal Cardiotocography.

22. Van Bogaert LJ, Misra A. Neonatal outcome after caesarean birth for fetal distress and/or meconium staining in a South African rural setting. J Obstetr and Gynaec. 2008 Jan 1;28(1):56-9.

23. Najmi RS. Justification of caesarean section for fetal distress. JPMA. 1997 Oct;47(10):250-2.

24. Walker J. Fetal distress.Am J Obstet Gynecol 1959;77:948.

25. Priyadharshini M, Panicker S. Meconium stained liquor and its fetal outcome-retrospective study. IOSR-JDMS. 2013;6(2):27-31

26. Liston R, Crane J, Hamilton E, Hughes $\mathrm{O}$ et al. Fetal health surveillance in labour. J Obstet Gynaecol Can. 2002 Mar;24(3):250-76.

27. Katz M, Meizner I, Mazor M, Insler V. Fetal heart rate patterns and scalp blood $\mathrm{pH}$ as predictors of fetal distress. Israel J med sci. 1981 Apr;17(4):260-5.

28. Bakr AF, Al-Abd M, Karkour T. Fetal pulse oximetry and neonatal outcome: A study in a developing country. $J$ perinato. $2005 \operatorname{Dec} 1 ; 25(12): 759$.

29. Gholitabar M, Ullman R, James D, Griffiths M. Caesarean section: summary of updated NICE guidance. BmJ. 2011 Nov 23;343:d7108.
30. Kaur J, Kaur K. Conditions behind fetal distress. Annals of Biolog Resea. 2012;3(10):4845-51.

CONFLICT OF INTEREST: Authors declare no conflict of interest

GRANT SUPPORT AND FINANCIAL DISCLOSURE: NIL

\section{AUTHOR'S CONTRIBUTION}

Following authors have made substantial contributions to the manuscript as under

Hussain SS: Conceived, Concept, Data Collection Results.

Fatima SS: Literature Review.

Shafqat T: Statiscal analysis.

Rasool F: Biblography.

Authors agree to be accountable for all aspects of the work in ensuring that questions related to the accuracy or integrity of any part of the work are appropriately investigated and resolved. 